\title{
Selected Topics in Biophotonics: Photodynamic Therapy and Optical Micromanipulation for Biophotonics
}

The present special section entitled "Selected Topics in Biophotonics: Photodynamic Therapy and Optical Micromanipulation for Biophotonics" comprises invited review papers and contributed papers from the summer school Biophotonics'09 as well as contributed papers within this general scope.

\section{Motivation and Purpose of Biophotonics Graduate Schools}

Over the past decade, lasers, optical methods, and instruments based on light interaction with tissues have emerged as powerful techniques for medical diagnostics, monitoring wide spectra of tissue function, and pathology. In biophysics and biology, optical sensing and manipulation of cells have strengthened understanding of basic cell function. Together with improved laser therapeutic techniques, optical sensing and cell manipulation form the basis for the increased interest in biophotonics. Throughout Europe, the U.S., and the rest of the world, major research centers are highly active in this field that in a broad sense may be labelled biophotonics. Therefore, education within this area is becoming increasingly important.

The main purpose with the biennial graduate summer school is to provide education within biophotonics for students and young scientists at the highest international level. Our aim is to attract internationally renowned researchers as lecturers who would attract the most talented young researchers worldwide in the field of biophotonics.

\section{Format of the Biophotonics Graduate Summer School}

The school mainly targets graduate students and postdoctoral fellows. The format of the school is a combination of lectures and student poster presentations, with time between lectures for discussions and exchange of scientific ideas. The lecturers cover one topic in a full session comprised of four lectures, which thoroughly covers the basics and state of the art of each topic. On one hand, this choice limits the number of topics taught at each school. On the other hand, the topics selected for the schools are covered in detail. Therefore, the range of topics taught will change from school to school.

An important feature of the school format is that students and lecturers spend the entire week together, which provides excellent opportunities for the exchange of scientific ideas, networking, and socializing.

The 4th International Graduate Summer School Biophotonics'09 covered the basics of lasers as well as supercontinuum sources and their application in medicine, tissue optics, photodynamic therapy, optical tweezers and their applications in biophotonics, optical biosensors, diffuse optical and molecular imaging, fluorescence nanoscopy, and optical coherence tomography. ${ }^{1}$

\section{Special Section in the Journal of Biomedical Optics}

We are pleased to introduce the contributions to this special section on "Selected Topics in Biophotonics: Photodynamic Therapy and Optical Micromanipulation for Biophotonics" comprised of two invited tutorial papers and 14 contributed papers, mainly from the participants of the school but also from other researchers in the field. Not all the contributions are strictly covered by the title of the special section, but all of the contributions reflect the core topics of the school and span the fields of biomedical optics and biophotonics. The two invited review papers are:

- Katarina Svanberg, Niels Bendsoe, Johan Axelsson, Stefan Andersson-Engels, and Sune Svanberg, "Photodynamic therapy: superficial and interstitial illumination."

- David J. Stevenson, Frank Gunn-Moore, and Kishan Dholakia, "Light forces the pace: optical manipulation for biophotonics."

These papers from lecturers at the school are tutorial in character and provide an excellent background to the fields of photodynamic therapy and light forces in biophotonics, respectively. The two invited review papers complement two previous tutorial papers on diffuse optics ${ }^{2}$ and molecular imaging $^{3}$ published in the special section from the previous school. These papers all belong to a planned series of tutorial review papers from each biennial school that provide high-level educational material for the benefit of the scientific community and, in addition, fulfill our own motivation for creating the school in the first place.

Following the two invited review papers, we have organized the contributed papers according to their main topic starting with papers categorized as contributions within micromanipulation in biophotonics using light forces. The first of these concerns how red blood cells rotate when optically trapped. This is explained by the shape anisotropy of the cells. Next, Aabo et al. investigate the short- and long-term effects of optical trapping at a wavelength of $1070 \mathrm{~nm}$. Their results indicate that there is no threshold for photodamage, but that cell growth was increasingly inhibited with power and accumulated total dose. The next two papers both concern optical transient transfection, with violet and femtosecond lasers, respectively. Violet light transfection is shown for the first time, in what is believed to be a major step toward a compact and affordable system for optical transfection. Femtosecond pulses were used to optimize optical transfection in mammalian stem cell differentiation. This group of papers is concluded with a paper describing in vivo carotid artery closure using functionalized gold nanorods as a specific absorber.

The next set of papers concerns imaging on a microscopic level including optical coherence tomography (OCT). 
In the first of these articles Rommel et al. manipulate the refractive index to enhance the contrast in digital holography of cellular structures. Optical coherence tomography is then described as a tool potentially meeting the clinical need to enable guiding epicardial cardiac radiofrequency ablation therapy. The next paper concerns the complex dependence of the frequency shift in Doppler spectral domain OCT. Walter et al. introduce a novel method to extend the limited velocity detection range by taking the signal power decrease due to fringe washout into account. This is followed by a paper from Winkler et al. presenting a software package that automatically identifies potentially diseased areas to enable efficient utilization of 3D-OCT for longitudinal studies for following disease progression. The last OCT paper presents a heterodyne detection technique to enhance the signal from retinal neuron flickering, providing a novel tool for functional imaging. This set of papers ends with an evaluation of the potential of confocal microscopy to noninvasively determine epidermal hyperplasia in mouse models.

The special section finishes with three articles on different topics. The first of the contributions deals with optical mammographic tomography compensating for an angled chest wall in the reconstruction. The next paper presents a Raman spectroscopic study of an antimalaria drug, providing insight into the drug mechanism. Lastly Schelb et al. illustrate a fluorescence excitation method to be used with monolithically integrated all-polymer chips.

\section{Acknowledgments}

The editors would first like to thank all the lecturers and participants of the summer school Biophotonics'09 for making the school a very fruitful and positive experience, the authors of all papers in this special section for their excellent contributions, and the many reviewers around the world who provided high-quality reviews of the manuscripts. Your dedicated efforts have made this high-quality special section possible. The editors would also like to thank the JBO publication staff, Rita Davis and Karolyn Labes in particular, for their invaluable support and prompt assistance in helping meet the JBO standards. Finally, the editors would like to thank Prof. Bruce Tromberg, past Editor-in-Chief, and Prof. Lihong Wang, Editor-in-Chief, for their never-ending support and stimulating encouragements during the entire process of making this special section.

\section{References}

1. Biophotonics '09 Website, (2009) 〈www.biop.dk/biophotonics09/>.

2. S. L. Jacques and B. W. Pogue, "Tutorial on diffuse light transport," J. Biomed. Opt. 13(4), 041302 (2008).

3. E. M. Sevick-Muraca and J. C. Rasmussen, "Molecular imaging with optics: primer and case for near-infrared fluorescence techniques in personalized medicine," J. Biomed. Opt. 13(4), 041303 (2008).

Stefan Andersson-Engels, Ph.D. Lund University

Peter E. Andersen, Ph.D. Technical University of Denmark

Special Section Guest Editors 